\title{
A method of feature selection in the aspect of specific identification of radar signals
}

\author{
J. DUDCZYK* \\ WB Electronics S.A., 129/133 Poznańska St., 05-850 Ożarów Mazowiecki, Poland
}

\begin{abstract}
This article presents an important task of classification, i.e. mapping surfaces which separate patterns in feature space in the scope of radar emitter recognition (RER) and classification. Assigning a tested radar to a particular class is based on defining its location from the discriminating areas. In order to carry out the classification process, it is necessary to define metrics in the feature space as it is essential to estimate the distance of a classified radar from the centre of the class. The method presented in this article is based on extraction and selection of distinctive features, which can be received in the process of specific emitter identification (SEI) of radar signals, and on the minimum distance classification. The author suggests a RER system which consists of a few independent channels. The task of each channel is to calculate the distance of the tested radar from a given class and finally, set the correct identification coefficient for each recognized radar. Thus, a multichannel system with independent distance measurement is obtained, which makes it possible to recognize particular radar copies. This system is implemented in electronic intelligence (ELINT) system and tested in real battlefield conditions.
\end{abstract}

Key words: radar emitter recognition (RER), specific emitter identification (SEI), minimum distance classification, ELINT system.

\section{Introduction}

In the theory of radar signals recognition and their emission sources, the main task is to set distinctive patterns of such signals and work out methods for their distinguishing $[1,2]$. In the literature, terms such as source emission patterns separating surfaces in the measurable features space are widely used to describe pattern recognition and pattern classification $[3,4,5]$. Taking into account the fact above, radar emitter recognition and classification is based on defining the location of the emission source from the above separating surfaces. Here, it is essential to indicate a very significant fact, namely, in order to set a distinctive radar signal pattern, radar metrics needs to be defined in the measurable feature space of this signal; only after that the pattern vector of such a signal in the database can be designed $[1,6,7]$. However, what needs to be taken into account is the specificity of such a database for ELINT systems and electronic warfare (EW), $[8,9,10]$. The specificity of such a database is not the subject of the content of this article. Radar metrics is based on a distinctive radar signal pattern used to calculate the distance of this pattern from the centre of the class. Classification in this case is based on calculation of the distance of the recognized radar pattern from the centre of the class and classifying it to the class with the smallest distance. In order for this problem of radar signal classification to be solvable, it is necessary to define the class of a radar. Defining classes is not only setting the expected average values of basic measurable parameters of a radar signal, i.e., radio frequency (RF), pulse width $(\mathrm{PW})$ and pulse repetition interval (PRI). This process also requires defining variance and correlation of these features [11]. The second significant element of the classification pro-

*e-mail: j.dudczyk@wb.com.pl cedure is the problem to define how to estimate the distance of a tested radar emitter signal from the centre of the class taking into consideration variance and correlation of vector's features. The RER method also provides a solution when the features of radar patterns are not linearly separable. RER method bases on the analysis of basic measurable parameters of the radar signal (such as RF, PW, PRI) as result of which it is possible to extract additional distinctive features. The RER process is called specific emitter identification (SEI).

Additionally extracted distinctive features, which are received in the process of RER, may be a product of out-of-band radiation of radar devices [12]. These features may be of fractal type, which is presented in the works $[13,14,15]$. The received features may also be a product of inter-pulse modulation [16] and intrapulse analysis of a radar signal [17]. Of course, there are more complicated approaches, which offer effective methods for solving the classification task (i.e. mapping separating surfaces). These are based on solving the linear approximation task recurrently, using gradient methods and nonlinear approximation [18], nonlinear approximation of random function [19] and other methods for adaptive regression splines, classification and approximation [20,21]. This is a typical solution for identification systems such as perceptrons or artificial neural network (ANN), e.g., support vector machine networks (SVM) [22] using Widrow-Hoff learning algorithms, Adaline ANN or the method based on back-propagating errors and neural network classifier with low discrepancy optimization $[23,24]$. Also, the Fourier transform has been widely used in radar signal and image processing. Another work [25] presents joint time-frequency (JTF) domain analysis as a useful tool for improving radar signal and image processing for time-and frequency-varying cases. In [26], quadratic time-frequency analysis and sequential recognition for SEI is presented. Another approach based on three-dimensional distribution feature is 
presented in [27]. Radar emitter signal identification based on hierarchical agglomerative clustering is analyzed in [28]. Vector neural network (VNN) with a supervised learning algorithm suitable for signal classification is very useful for emitter identification and radar classification process, as shown in $[29,30]$.

\section{Multi-channel recognition system with an independent distance measurement}

During the measurement procedure, radar signals in the form of basic vector parameters $\mathbf{V}_{\mathrm{B}}$ are defined according to (1), where $n_{w}$ is the number of pulses in samples which are classified to the analysis and $k$ is the number of pulses in a measurement sample. As a general rule, in one sample there are 5000 pulses while a superheterodyne ELINT receiver is used to record a package of radar signals [12].

$$
\mathbf{V}_{\mathrm{B}}=\left[\frac{1}{n_{w}} \sum_{k=1}^{n_{w}} \operatorname{RF}(k), \frac{1}{n_{w}} \sum_{k=1}^{n_{w}} \operatorname{PW}(k), \frac{1}{n_{w}} \sum_{k=1}^{n_{w}} \operatorname{PRI}(k)\right]
$$

The suggested RER system consists of independent channels. Each of the channels is assigned to one class of the recognized radars. The main task of every single channel is to estimate the distance of a tested radar signal from its class. The distance of the tested radar signal (defined by features' values of its basic measurable parameters) $\mathbf{V}_{\mathrm{B}} \in R^{n}$ from a particular class with $i$ index marked as $d_{i}\left(\mathbf{V}_{\mathrm{B}}\right)$. It should be mentioned that it is the value estimated as a distance from " $\mathbf{V}_{\mathrm{B}}$ point" to the centre of i-class. As a result of the procedure above, the measurement of the distance received from channels is the base for recognition. The radar is recognized as belonging to a particular class with $w$ index, if $d_{w}(\mathbf{u})=\min \left\{d_{1}\left(\mathbf{V}_{\mathrm{B} 1}\right), d_{2}\left(\mathbf{V}_{\mathrm{B} 2}\right)\right\}$. A significant problem which comes out in the method above concerns the rule of choosing a decision, which is based on defining values of different $d_{1}\left(\mathbf{V}_{\mathrm{B}}\right)$ functionals. This is a problem which concerns mathematical reasoning [31]. In order to solve this problem it is assumed that $\mathbf{V}_{\mathrm{B} k}$ is a random variable which defines features' values of elements of a radar class with $k$ index and $\mathrm{E} d_{j}\left(\mathbf{V}_{\mathrm{B} k}\right)$ is an expected value of the distance $d_{j}$ for the radar class with $k$ index

\section{Defining the distance on impartiality condition}

To start with, a particular class $\mathrm{G}$ of radars is chosen for which the centre of the class $\overline{\mathbf{V}}_{\mathrm{B}}=\mathrm{E}\left(\mathbf{V}_{\mathrm{B}}\right)$ is defined and covariance matrix of the vector features $\mathbf{V}_{\mathrm{B}}$ from $\mathrm{G}$ class, according to (2), where $\mathrm{T}$ is a sign of matrix transposition.

$$
\mathbf{R}=\mathrm{E}\left(\mathbf{V}_{\mathrm{B}}-\overline{\mathbf{V}}_{\mathrm{B}}\right)\left(\mathbf{V}_{\mathrm{B}}-\overline{\mathbf{V}}_{\mathrm{B}}\right)^{T}
$$

The eigenvalues denoted as $\lambda_{k}$ and orthogonal eigenvectors denoted as $\mathbf{t}_{k}$ for each covariance matrix $\mathrm{R}$ are calculated according to (3) and (4).

$$
\begin{aligned}
\mathbf{R} \mathbf{t}_{k}=\lambda_{k} \mathbf{t}_{k} \text { where } k=1,2, \ldots, n \\
\mathbf{t}_{i}^{T} \mathbf{t}_{j}= \begin{cases}1 & \text { if } \quad i=j \\
0 & \text { if } \quad i \neq j\end{cases}
\end{aligned}
$$

In order to define the distance of $\mathbf{V}_{\mathrm{B}}$ vector from $\mathrm{G}$ class, Karhunen-Loeve (K-L) transform with transformation matrix $\mathrm{T}$ are used. The lines of the T matrix are the $\mathrm{R}$ matrix's eigenvectors, according to (5), where for $\mathbf{V}_{\mathrm{B}}$ argument there is a result in a form of a transformation vector $\mathbf{T} \mathbf{V}_{\mathrm{B}}$ according to (6).

$$
\mathbf{T}=\left[\begin{array}{c}
\mathbf{t}_{1}^{T} \\
\mathbf{t}_{2}^{T} \\
\cdots \\
\mathbf{t}_{n}^{T}
\end{array}\right]
$$

$$
\mathbf{T} \mathbf{V}_{\mathrm{B}}=\left[\begin{array}{c}
\mathbf{t}_{1}^{T} \mathbf{V}_{\mathrm{B}} \\
\mathbf{t}_{2}^{T} \mathbf{V}_{\mathrm{B}} \\
\cdots \\
\mathbf{t}_{n}^{T} \mathbf{V}_{\mathrm{B}}
\end{array}\right]
$$

Taking into consideration the fact that the base for estimation of the distance of $\mathbf{V}_{B}$ vector feature from $G$ class is the difference $\mathbf{r}=\mathbf{V}_{\mathrm{B}}-\overline{\mathbf{V}}_{\mathrm{B}}$, the next step is to define $\mathrm{K}$-L transform of the estimated difference, according to (7).

$$
\mathbf{y}=\left[\begin{array}{c}
y_{1} \\
y_{2} \\
\cdots \\
y_{n}
\end{array}\right]=\mathbf{T r}=\left[\begin{array}{c}
\mathbf{t}_{1}^{T} \mathbf{r} \\
\mathbf{t}_{2}^{T} \mathbf{r} \\
\ldots \\
\mathbf{t}_{n}^{T} \mathbf{r}
\end{array}\right]
$$

The decorrelation process of the vector's features' co-ordinates according to (8) and the normalization process of dispersion of co-ordinates' values according to (9) results in a correlation matrix equal to an identity matrix according to (10).

$$
\begin{aligned}
& \mathrm{E}\left(\mathbf{y} \mathbf{y}^{T}\right)=\mathrm{E}[\mathbf{T r}][\mathbf{T r}]^{T}=\mathbf{T E}\left(\mathbf{r} \mathbf{r}^{T}\right) \mathbf{T}^{T}=\mathbf{T R} \mathbf{T}^{T}= \\
& =\left[\begin{array}{c}
\mathbf{t}_{1}^{T} \\
\mathbf{t}_{2}^{T} \\
\ldots \\
\mathbf{t}_{n}^{T}
\end{array}\right] \mathbf{R}\left[\mathbf{t}_{1}, \mathbf{t}_{2}, \ldots, \mathbf{t}_{n}\right]=\left[\begin{array}{c}
\mathbf{t}_{1}^{T} \\
\mathbf{t}_{2}^{T} \\
\ldots \\
\mathbf{t}_{n}^{T}
\end{array}\right] \cdot\left[\mathbf{R} \mathbf{t}_{1}, \mathbf{R} \mathbf{t}_{2}, \ldots, \mathbf{R} \mathbf{t}_{n}\right]= \\
& =\left[\begin{array}{cccc}
\lambda_{1} \mathbf{t}_{1}^{T} \mathbf{t}_{1} & \lambda_{2} \mathbf{t}_{1}^{T} \mathbf{t}_{2} & \ldots & \lambda_{n} \mathbf{t}_{1}^{T} \mathbf{t}_{n} \\
\lambda_{1} \mathbf{t}_{2}^{T} \mathbf{t}_{1} & \lambda_{2} \mathbf{t}_{2}^{T} \mathbf{t}_{2} & \ldots & \lambda_{n} \mathbf{t}_{2}^{T} \mathbf{t}_{n} \\
\ldots & \ldots & \ldots & \ldots \\
\lambda_{1} \mathbf{t}_{n}^{T} \mathbf{t}_{1} & \lambda_{2} \mathbf{t}_{n}^{T} \mathbf{t}_{2} & \ldots & \lambda_{n} \mathbf{t}_{n}^{T} \mathbf{t}_{n}
\end{array}\right]= \\
& =\operatorname{diag}\left(\lambda_{1}, \lambda_{2}, \ldots, \lambda_{n}\right)=\boldsymbol{\Lambda}
\end{aligned}
$$




$$
\begin{aligned}
& \mathbf{z}=\left[\begin{array}{c}
z_{1} \\
z_{2} \\
\cdots \\
z_{n}
\end{array}\right]=\left[\begin{array}{c}
\frac{y_{1}}{\sqrt{\lambda_{1}}} \\
\frac{y_{2}}{\sqrt{\lambda_{2}}} \\
\cdots \\
\frac{y_{n}}{\sqrt{\lambda_{n}}}
\end{array}\right] \\
& \mathrm{E}\left(\mathbf{z z}^{\prime}\right)=E\left[\begin{array}{cccc}
z_{1} z_{1} & z_{1} z_{2} & \ldots & z_{1} z_{n} \\
z_{2} z_{1} & z_{2} z_{2} & \ldots & z_{2} z_{n} \\
\ldots & \ldots & \ldots & \ldots \\
z_{n} z_{1} & z_{n} z_{2} & . . & z_{n} z_{n}
\end{array}\right]= \\
& =\mathrm{E}\left[\begin{array}{cccc}
\frac{y_{1} y_{1}}{\lambda_{1}} & \frac{y_{1} y_{2}}{\sqrt{\lambda_{1} \lambda_{2}}} & \ldots & \frac{y_{1} y_{n}}{\sqrt{\lambda_{1} \lambda_{n}}} \\
\frac{y_{2} y_{2}}{\sqrt{\lambda_{2} \lambda_{1}}} & \frac{y_{2} y_{2}}{\lambda_{2}} & \ldots & \frac{y_{2} y_{n}}{\sqrt{\lambda_{2} \lambda_{n}}} \\
\ldots & \ldots & \ldots & \ldots \\
\frac{y_{n} y_{2}}{\sqrt{\lambda_{n} \lambda_{1}}} & \frac{y_{n} y_{2}}{\sqrt{\lambda_{n} \lambda_{2}}} & \ldots & \frac{y_{n} y_{n}}{\lambda_{n}}
\end{array}\right]=\operatorname{diag}(1,1, \ldots, 1)
\end{aligned}
$$

The $d\left(\mathbf{V}_{\mathrm{B}}\right)$ distance of $\mathbf{V}_{\mathrm{B}}$ vector from $\mathrm{G}$ class is defined according to (11), where $d\left(\mathbf{V}_{\mathrm{B}}\right)$ functional defines the distance from $\mathbf{V}_{B}$ features' vector of a tested radar to the $\overline{\mathbf{V}}_{B}$ centre as a Mahalanobis distance.

$$
\begin{aligned}
& d\left(\mathbf{V}_{\mathrm{B}}\right)=\mathbf{z}^{T} \mathbf{z}=\sum_{k=1}^{n} z_{k}^{2}=\sum_{k=1}^{n} \frac{y_{k}^{2}}{\lambda_{k}}=\sum_{k=1}^{n} \frac{\left(\mathbf{t}_{k}^{T} \mathbf{r}\right)^{2}}{\lambda_{k}}= \\
& =\sum_{k=1}^{n}\left(\mathbf{r}^{T} \mathbf{t}_{k}\right) \frac{1}{\lambda_{k}}\left(\mathbf{t}_{k}^{T} \mathbf{r}\right)= \\
& =\mathbf{r}^{T}\left[\mathbf{t}_{1}, \mathbf{t}_{2}, \ldots, \mathbf{t}_{n}\right]\left[\begin{array}{cccc}
\lambda_{1}^{-1} & 0 & \ldots & 0 \\
0 & \lambda_{2}^{-1} & \ldots & 0 \\
\ldots & \ldots & \ldots & \ldots \\
0 & 0 & \ldots & \lambda_{n}^{-1}
\end{array}\right]\left[\begin{array}{c}
\mathbf{t}_{1}^{T} \\
\mathbf{t}_{2}^{T} \\
\ldots \\
\mathbf{t}_{n}^{T} \\
\overline{T^{T}}
\end{array}\right] \mathbf{r}= \\
& =\left(\mathbf{V}_{\mathrm{B}}-\overline{\mathbf{V}}_{\mathrm{B}}\right)^{T} \mathbf{T}^{T} \boldsymbol{\Lambda}^{-1} \mathbf{T}\left(\mathbf{V}_{\mathrm{B}}-\overline{\mathbf{V}}_{\mathrm{B}}\right)=\left(\mathbf{V}_{\mathrm{B}}-\overline{\mathbf{V}}_{\mathrm{B}}\right)^{T} \mathbf{R}^{-1}\left(\mathbf{V}_{\mathrm{B}}-\overline{\mathbf{V}}_{\mathrm{B}}\right)
\end{aligned}
$$

As a result of averaging out the received functional according to (11), $E d(\mathbf{u})$ value is received according to (12).

$$
\begin{aligned}
& \mathrm{E} d\left(\mathbf{V}_{\mathrm{B}}\right)=\mathrm{E}\left[\left(\mathbf{V}_{\mathrm{B}}-\overline{\mathbf{V}}_{\mathrm{B}}\right)^{T} \mathbf{R}^{-1}\left(\mathbf{V}_{\mathrm{B}}-\overline{\mathbf{V}}_{\mathrm{B}}\right)\right]=\mathrm{E}\left[\mathbf{r}^{T} \mathbf{T}^{T} \boldsymbol{\Lambda}^{-1} \mathbf{T r}\right]= \\
& =\mathrm{E}\left[y^{T} \boldsymbol{\Lambda}^{-1} y\right]=\mathrm{E}\left\{\mathbf{y}^{T}\left[\begin{array}{cccc}
\lambda_{1}^{-1} & 0 & \ldots & 0 \\
0 & \lambda_{2}^{-1} & \ldots & 0 \\
\ldots & \ldots & \ldots & \ldots \\
0 & 0 & \ldots & \lambda_{n}^{-1}
\end{array}\right] \mathbf{y}\right\}= \\
& =\mathrm{E} \sum_{k=1}^{n} \frac{y_{k}^{2}}{\lambda_{k}}=\sum_{k=1}^{n} \frac{\mathrm{E} y_{k}^{2}}{\lambda_{k}}=n
\end{aligned}
$$

The analysis above makes it possible to use the definition of distance $d_{i}\left(\mathbf{V}_{\mathrm{B}}\right)$ for the channel with index $i \in\{1,2, \ldots, L\}$ according to (13), where $\overline{\mathbf{V}}_{\mathrm{B} i}$ is the expected value of $\mathbf{G}_{i}$ features' class vector, while $\mathbf{R}_{i}=\mathrm{E}\left(\mathbf{V}_{\mathrm{B} i}-\overline{\mathbf{V}}_{\mathrm{B} i}\right)\left(\mathbf{V}_{\mathrm{B} i}-\overline{\mathbf{V}}_{\mathrm{B} i}\right)^{T}$ is the covariance matrix of $\mathbf{G}_{i}$ class. It is also necessary to mention that $d_{i}$ functionals, which are defined by (14), where $\mathbf{V}_{\mathrm{B} i}$ is a random variable which defines the values of $\mathbf{G}_{i}$ class elements, fulfill the impartiality condition $\mathrm{E} d_{1}\left(\mathbf{V}_{\mathrm{B} 1}\right)=\mathrm{E} d_{2}\left(\mathbf{V}_{\mathrm{B} 2}\right)$.

$$
\begin{gathered}
d_{i}\left(\mathbf{V}_{\mathrm{B}}\right)=\left(\mathbf{V}_{\mathrm{B}}-\overline{\mathbf{V}}_{\mathrm{B} i}\right)^{T} \mathbf{R}_{i}^{-1}\left(\mathbf{V}_{\mathrm{B}}-\overline{\mathbf{V}}_{\mathrm{B} i}\right) \\
\mathbf{E} d_{i}\left(\mathbf{V}_{\mathrm{B} i}\right)=n \quad i \in\{1,2, \ldots, L\}
\end{gathered}
$$

In spite of $\mathbf{R}_{i}$ parameter diversity, the values of the distance estimated according to (13) can be comparable and are the base to define the class of the nearest recognized radar emitter signal.

\section{The process of learning}

The presented radar recognition process is supposed to be preceded by the process of learning. This process aims at estimation of the expected value of class patterns according to (15), estimation of covariance matrix of classes' patterns according to (16) for each of the collections $\mathbf{G}_{i}$ class patterns and defining eigenvalues of covariance matrix $\mathbf{R}_{i}$ and belonging to them, orthogonal eigenvectors according to $(17,18)$.

$$
\begin{gathered}
\overline{\mathbf{V}}_{\mathrm{B} i}=\frac{1}{\left\|G_{i}\right\|} \sum_{\mathbf{x} \in G_{i}} \mathbf{x} \quad i \in\{1,2, \ldots, L\} \\
\mathbf{R}_{i}=\frac{1}{\left\|G_{i}\right\|} \sum_{\mathbf{x} \in G_{i}}\left(\mathbf{x}-\overline{\mathbf{V}}_{\mathrm{B} i}\right)\left(\mathbf{x}-\overline{\mathbf{V}}_{\mathrm{B}_{i}}\right)^{T} \quad i \in\{1,2, \ldots, L\} \\
\mathbf{R}_{i} \mathbf{t}_{k}^{(i)}=\lambda_{k}^{(i)} \mathbf{t}_{k}^{(i)} \quad k \in\{1,2, \ldots, n\} \\
\mathbf{T}_{i}=\left[\begin{array}{c}
\left(\mathbf{t}_{1}^{(i)}\right)^{T} \\
\left(\mathbf{t}_{2}^{(i)}\right)^{T} \\
\ldots \\
\left(\mathbf{t}_{n}^{(i)}\right)^{T}
\end{array}\right] \quad i \in\{1,2, \ldots, L\}
\end{gathered}
$$

The analysis requires the assumption that covariance matrices might be nonsingular. Sometimes tests show that covariance matrix can be singular. As a result, it is not possible to normalize coordinates of $\mathrm{K}-\mathrm{L}$ transform according to (8). Thus, there is a need to normalize coordinates of $\mathrm{K}-\mathrm{L}$ transform according to (19).

$$
\mathbf{z}_{k}=\left\{\begin{array}{ccc}
\frac{y_{k}}{\sqrt{\lambda_{k}}} & \text { if } & \lambda_{k} \neq 0 \\
0 & \text { if } & \lambda_{k}=0
\end{array}\right.
$$

This causes that $d\left(\mathbf{V}_{\mathrm{B}}\right)$ distance of the $\mathbf{V}_{\mathrm{B}}$ vector from $\mathrm{G}$ class, defined according to (11), is modified to the form of (20), where $\mathrm{p}$ is the number of non-zero eigenvalues of co- 
variance matrix, $\mathbf{R}^{+}$is Moore-Penrose inverse of covariance matrix, according to the relation $\mathbf{R}^{+}=\mathbf{T}^{T} \mathbf{M T}$, where $\mathbf{M}$ is the matrix with (21).

$$
\begin{aligned}
& d\left(\mathbf{V}_{\mathrm{B}}\right)=\mathbf{z}^{T} \mathbf{z}=\sum_{k=1}^{p} z_{k}^{2}=\sum_{k=1}^{p} \frac{y_{k}^{2}}{\lambda_{k}}=\sum_{k=1}^{p} \frac{\left(\mathbf{t}_{k}^{T} \mathbf{r}\right)^{2}}{\lambda_{k}}= \\
& =\sum_{k=1}^{p}\left(\mathbf{r}^{T} \mathbf{t}_{k}\right) \frac{1}{\lambda_{k}}\left(\mathbf{t}_{k}^{T} \mathbf{r}\right)= \\
& =\mathbf{r}^{T}\left[\mathbf{t}_{1} \ldots \mathbf{t}_{p} \ldots \mathbf{t}_{n}\right]\left[\begin{array}{cccc}
\lambda_{1}^{-1} & 0 & \ldots & 0 \\
0 & \lambda_{p}^{-1} & \ldots & 0 \\
\ldots & \ldots & \ldots & \ldots \\
0 & 0 & \ldots & 0
\end{array}\right]\left[\begin{array}{c}
\mathbf{t}_{1}^{T} \\
\mathbf{t}_{p}^{T} \\
\ldots \\
\mathbf{t}_{n}^{T}
\end{array}\right] \mathbf{r}= \\
& =\left(\mathbf{V}_{\mathrm{B}}-\overline{\mathbf{V}}_{\mathrm{B}}\right)^{T} \mathbf{T}^{T} \mathbf{M T}\left(\mathbf{V}_{\mathrm{B}}-\overline{\mathbf{V}}_{\mathrm{B}}\right)= \\
& =\left(\mathbf{V}_{\mathrm{B}}-\overline{\mathbf{V}}_{\mathrm{B}}\right)^{T} \mathbf{R}^{+}\left(\mathbf{V}_{\mathrm{B}}-\overline{\mathbf{V}}_{\mathrm{B}}\right) \\
& \mathbf{M}=\left[\begin{array}{cccc}
\lambda_{1}^{-1} & 0 & \ldots & 0 \\
0 & \lambda_{p}^{-1} & \ldots & 0 \\
\ldots & \ldots & \ldots & \ldots \\
0 & 0 & \ldots & 0
\end{array}\right]
\end{aligned}
$$

The analysis above makes it possible to create a scheme of recognition system with the use of $\mathrm{K}-\mathrm{L}$ transform, which is presented in Fig. 1.

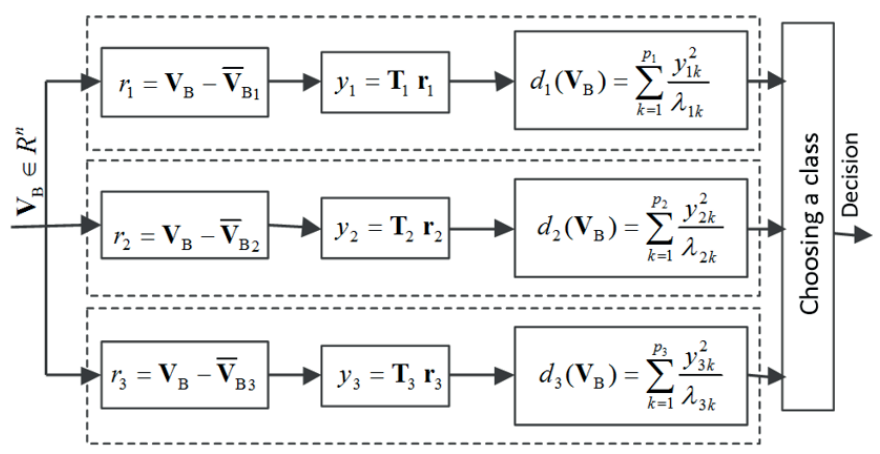

Fig. 1. A scheme of a triple-channel RER system based on K-L transform

\section{Calculation results and received RER results}

The defined distance $d_{i}\left(\mathbf{V}_{\mathrm{B}}\right)$ for particular recognition channels with a coefficient $i \in\{1,2, \ldots, L\}$ according to (13) and modification of this distance according to (20), is a base for classification of the tested radar to an appropriate class. In order to compare the received results of RER method with other methods described in works $[7,12,13,15-17,32]$, correct identification coefficient (CIC) is set according to (22), where $n_{B-P}$ is the number of correct comparisons of basic features' vectors $\mathbf{V}_{B}$ to pattern vectors $\mathbf{V}_{\mathrm{P}}$ in a particular class, and $N$ is the number of all comparisons divided by the number of test collections.

$$
\mathrm{CIC}=\frac{n_{B-P}}{N}
$$

The number of $n_{B-P}$ correct comparisons is set according to (23), where $\gamma_{i}^{j}$ function assigns to a pair of vectors $\left(\mathbf{V}_{\mathrm{B}}^{i}, \mathbf{V}_{\mathrm{P}}^{j}\right)$ the value which equals ' 1 ' if $\mathrm{i}=\mathrm{j}$, or the value which equals ' 0 ' if $\mathrm{i} \neq \mathrm{j}$. The received CIC values are presented in Table 4 .

$$
n_{B-P}=\sum_{i=1}^{I} \sum_{j=1}^{J} \gamma^{i j}
$$

During the test, the total comes to a few hundreds of radar signals recorded in collections with the length of 5000 pulses. A superheterodyne ELINT receiver was used in the measuring procedure. This receiver makes it possible to define value of RF with measurement accuracy $0.5 \mathrm{MHz}$ and value of PRI in the scope from $2 \mu \mathrm{s}$ to $20 \mathrm{~ms}$, with measurement accuracy $0.05 \mu \mathrm{s}$. The recordings came from a dozen or so radars of the same type. Three copies were chosen to further analysis and their measurable parameters were as follows: $\mathrm{RF}=1415 \mathrm{MHz}, \mathrm{PW}=11.2 \mu \mathrm{s}$ and the following PRI values i.e. $\mathrm{PRI}_{\min }=1400.96 \mu \mathrm{s}$, $\mathrm{PRI}_{\text {ave }}=1745.93 \mu \mathrm{s}$ and $\mathrm{PRI}_{\max }=2090.91 \mu \mathrm{s}$. The pulse repetition interval value (measured between the arrival times of two consecutive pulses on the level of the detection threshold) changes sequentially every pair of pulses, taking seven different PRI values. The complete cycle of value changes of PRI was as follows: $\mathrm{PRI}_{1}=2091 \mu \mathrm{s}, \mathrm{PRI}_{2}=1738 \mu \mathrm{s}, \mathrm{PRI}_{3}=1575 \mu \mathrm{s}$, $\mathrm{PRI}_{4}=1491 \mu \mathrm{s}, \quad \mathrm{PRI}_{5}=1447 \mu \mathrm{s}, \quad \mathrm{PRI}_{6}=1420 \mu \mathrm{s} \quad$ and $\mathrm{PRI}_{7}=1401 \mu \mathrm{s}$. This is a complete cycle of value changes of the PRI in a measurement sample. The method by which it was possible to receive a complete cycle of changes of PRI values is described in the works $[12,28]$. As a result of initial measurement data processing, $\mathbf{V}_{\mathrm{B}}$ vectors are received according to (1). On the basis of the received $\mathbf{V}_{B}$ vectors, pattern vectors $\mathbf{V}_{P}$ are also received. The method which is also used in this process is called the Holdout method. This method divides the available collection into two disjoint sets i.e. a set which is used to learn the classificator and a set to test the classificator. The usual division of the available data collection is as follows: $2 / 3$ of available data is in the learning set and $1 / 3$ is in the test set [12]. The identification process is carried out on the basis of the distance measurement with the use of the minimum-distance classification, according to the presented method. In order to assess the quality of the classification process the CIC index is defined. In the final process of identification, fast-decision identification algorithm (FDIA) of radar emission source is used in relational ELINT database (DB). The FDIA algorithm mentioned above is a three-stage parametrized by implementation of three different ways to define the similarity degree of the signal vector to the pattern in the database and by the possibility to define three-stage value of decision function. As a result this algorithm working in the classification process 
of radar types, the following CIC values are received i.e.: $88 \%$ and $92 \%$ for three different values of decision function. In case of identification of copies, the following CIC values are received: $26 \%, 47 \%$ and $63 \%$. The mechanism of FDIA algorithm and the received identification results are described in [7] in detail.

The received estimation results are presented in Tables 1-3 and illustrated in Figs. 2-4. Appropriately crossed columns and lines of each $\mathbf{V}_{\mathrm{B}}$ vector and radar patterns in the form of $\mathbf{V}_{\mathrm{P}}$ vectors, present the degree of their similarity defined by the distance value. The less value of this distance means the bigger similarity of $\mathbf{V}_{\mathrm{B}}$ vector to the pattern. Also, in Figs. 2-4 there are minimum values of the distance $d_{i}\left(\mathbf{V}_{\mathrm{B}}\right)$ marked with a red dotted ellipse in each $\mathbf{G}_{i}$ class. The received minimum

Table 1

The results of distance calculations for a radar copy No. 1 in L1 channel for 16 comparisons in G1 class

\begin{tabular}{|c|r|c|c|c|}
\hline \multirow{4}{*}{$\mathbf{d}_{1}\left(\mathbf{V}_{\mathrm{B}}-\mathbf{V}_{\mathrm{P}}\right)$} & \multicolumn{4}{|c|}{$\mathbf{V}_{\mathrm{B}}$ (for $\mathrm{G}_{1}$ class) } \\
\hline \multirow{3}{*}{$\begin{array}{c}\mathbf{V}_{\mathrm{P}} \\
\text { (for } \mathrm{G}_{1} \text { class) }\end{array}$} & 0,714 & 11,647 & 4,302 & 3,374 \\
\cline { 2 - 5 } & 11,821 & 0,798 & 3,567 & 6,995 \\
\cline { 2 - 5 } & 3,267 & 3,414 & 0,516 & 4,273 \\
\cline { 2 - 5 } & 5,278 & 6,728 & 4,178 & 0,612 \\
\hline
\end{tabular}

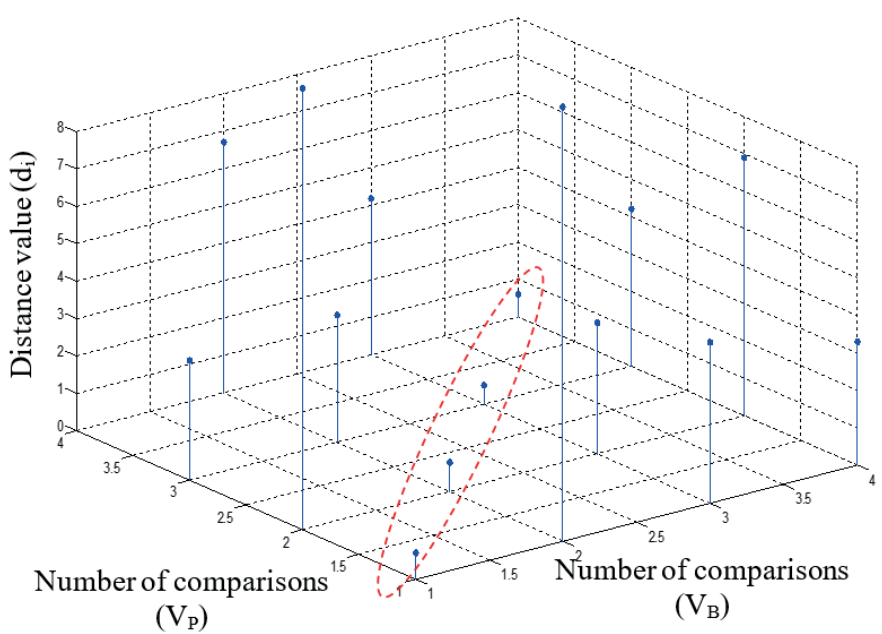

Fig. 2. 3D graphic illustration of the received values of distances in L1 channel for 16 comparisons of the radar copy No. 1 in G1 class

Table 2

The results of distance calculations for a radar copy No. 2 in L2 channel for 16 comparisons in G2 class

\begin{tabular}{|c|c|c|c|c|}
\hline \multirow{3}{*}{$\mathbf{d}_{2}\left(\mathbf{V}_{\mathrm{B}}-\mathbf{V}_{\mathrm{P}}\right)$} & \multicolumn{4}{|c|}{$\mathbf{V}_{\mathrm{B}}$ (for $\mathrm{G}_{2}$ class) } \\
\hline \multirow{3}{*}{$\begin{array}{c}\mathbf{V}_{\mathrm{P}} \\
\text { (for } \mathrm{G}_{2} \text { class) }\end{array}$} & 0,001 & 4,717 & 2,391 & 3,793 \\
\cline { 2 - 5 } & 4,732 & 0,022 & 11,207 & 2,721 \\
\cline { 2 - 5 } & 2,389 & 11,197 & 0,01 & 6,322 \\
\cline { 2 - 5 } & 4,796 & 2,717 & 6,329 & 0,008 \\
\hline
\end{tabular}

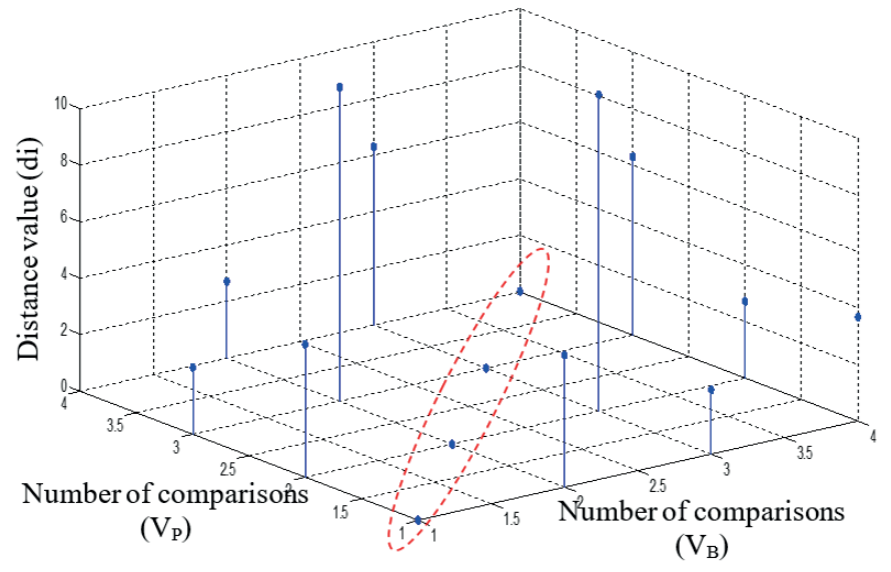

Fig. 3. 3D graphic illustration of the received distance values in L2 channel for 16 comparisons of the radar copy No. 2 in G2 class

Table 3

The results of distance calculations for a radar copy No. 3 in L3 channel for 16 comparisons in G3 class

\begin{tabular}{|c|r|r|r|r|}
\hline \multirow{4}{*}{$\mathbf{d}_{3}\left(\mathbf{V}_{\mathrm{B}}-\mathbf{V}_{\mathrm{P}}\right)$} & \multicolumn{4}{|c|}{$\mathbf{V}_{\mathrm{B}}$ (for $\mathrm{G}_{3}$ class) } \\
\hline \multirow{3}{*}{$\begin{array}{c}\mathbf{V}_{\mathrm{P}} \\
\text { (for } \mathrm{G}_{3} \text { class) }\end{array}$} & 0,349 & 11,105 & 5,705 & 3,401 \\
\cline { 2 - 5 } & 11,258 & 0,217 & 32,061 & 2,715 \\
\cline { 2 - 5 } & 5,804 & 32,012 & 0,249 & 17,058 \\
\cline { 2 - 5 } & 3,432 & 2,617 & 16,994 & 0,315 \\
\hline
\end{tabular}

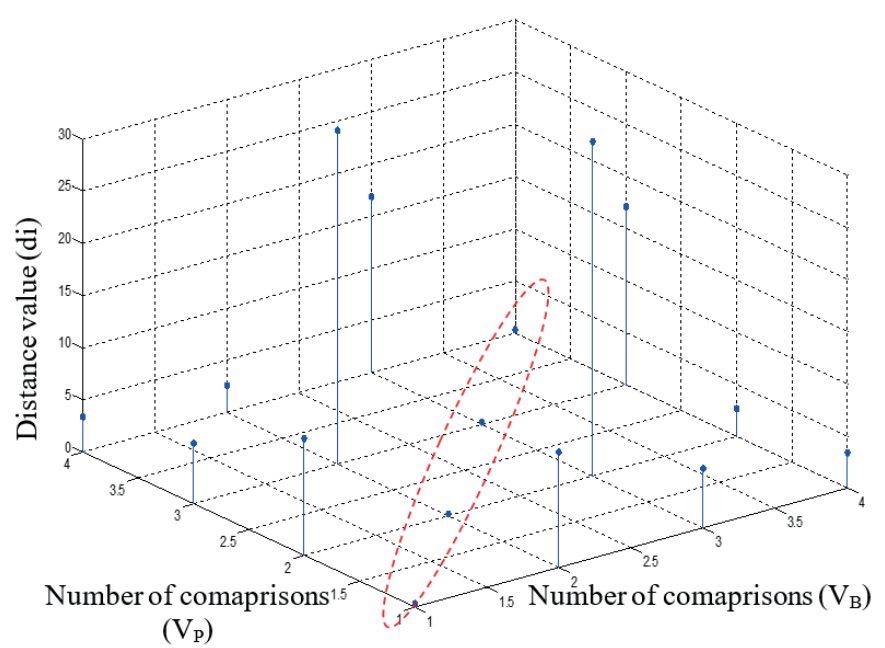

Fig. 4. 3D Graphic illustration of the received distance values in L3 channel for 16 comparisons of the radar copy No. 3 in G3 class

Table 4

CIC values of RER method for three recognized radar copies in $\mathrm{G} 1 \div \mathrm{G} 3$ classes

\begin{tabular}{|c|c|c|c|}
\hline CIC $_{\text {RER }}$ & $\mathbf{G}_{1}$ class & $\mathbf{G}_{2}$ class & $\mathbf{G}_{3}$ class \\
\hline $\mathrm{V}_{\mathrm{B}}$ & 0,234 & 0,194 & 0,264 \\
\hline $\mathrm{V}_{\mathrm{P}}$ & 0,534 & 0,614 & 0,498 \\
\hline
\end{tabular}




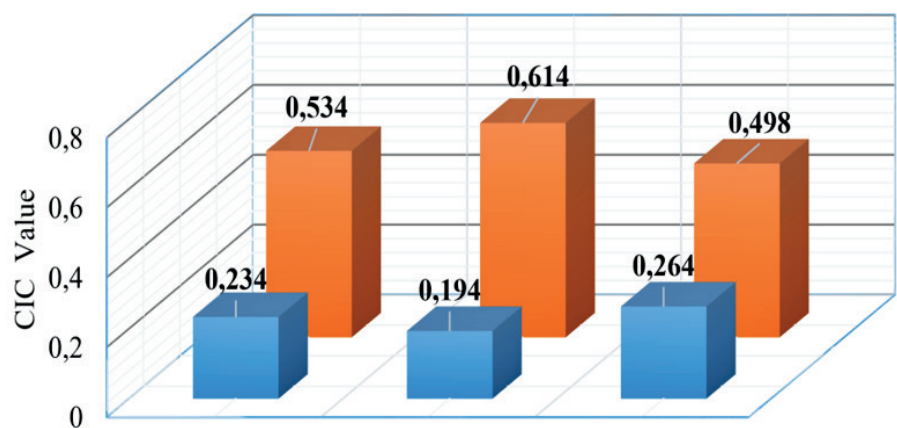

Fig. 5. Graphic illustration of CIC calculations for three radar copies in given classes $\mathrm{G} 1 \div \mathrm{G} 3$ of triple-channel RER system

distances show at the same time the number of correct comparisons of basic features' vectors to pattern vectors in the class. In each class $\left(\mathbf{G}_{1} \div \mathbf{G}_{3}\right) 16$ comparisons of these vectors are carried out and CIC is calculated. The results are presented in Table 4 and illustrated graphically in Fig. 5. According to the SEI methods listed in this section, the received RER results are as follows: the use of out-of-band radiation described in the work [12] the CIC value for RER is about $90 \%$. The method based on fractal features described in the work [15] the CIC value is $91.6 \%$ for Mahalanobis metric and 96.7\% for Euclidean and Hamming metrics. Very similar RER results are received in the work [13], where RER also bases on the analysis of fractal features. The method based on inter-pulse analysis described in the work [16] increases the CIC coefficient up to $70 \%$, and the method based on intrapulse analysis described in the work [17] makes it possible to receive RER results reaching 90\% level. Data modeling applied to RER and identification is presented in the work [32]. In this work the value of CIC equals $98 \%$. In the work [7], the fast identification algorithm for RER is presented. This algorithm is parametrized in three stages by implementation of three different ways to define the similarity degree of the signal vector to the pattern in the database. Based on this algorithm, the CIC value is $63 \%$.
In order to depict it clearly, CIC values received in other RER methods which appeared in this section are presented in Fig. 6.

It needs to be emphasized with full conviction that referring to the works above, during the RER procedure, the same recordings of a few hundred radar signals coming from the same type of radars are used. Only by this approach it is possible to compare the received results. It needs to be emphasized that the methods listed above, differ from each other as concerns the test procedure, the compilation level, calculation time and algorithm complexity. However, the main difference is that in the process of generating distinctive features, it is possible to achieve different distinctive features from a radar signal. In that way, a quasi-optimum radar signal pattern appeared.

\section{Conclusion}

The received measurement data have a significant influence on the specific identification process of radar, in which it is aimed to receive very high level of radar emitter signal identification. Ultimately, signal source identification which is $100 \%$, should be characterized by the maximization of explicitness of RER procedure. It is not a trivial matter to achieve such a result. It is also known that stochastic context-free grammars (SCFG) appear promising for the recognition and threat assessment of complex radar emitters in radar systems, but the computational requirements for learning their production rule probabilities can be very onerous [33]. As shown in [34], a self-organizing map and the maximum likelihood gamma mixture model classifier and adopted Bayesian formalism are too complicated for direct analytical use in automatic radar recognition. The presented RER method is realized on the basis of MatLab software package and received vectors are recorded in a dedicated database for ELINT system. The received CIC value indicates that there has been a noticeable rise in the radar signal correct identification. Comparing the received results of the identification process with other methods, it may be admitted that

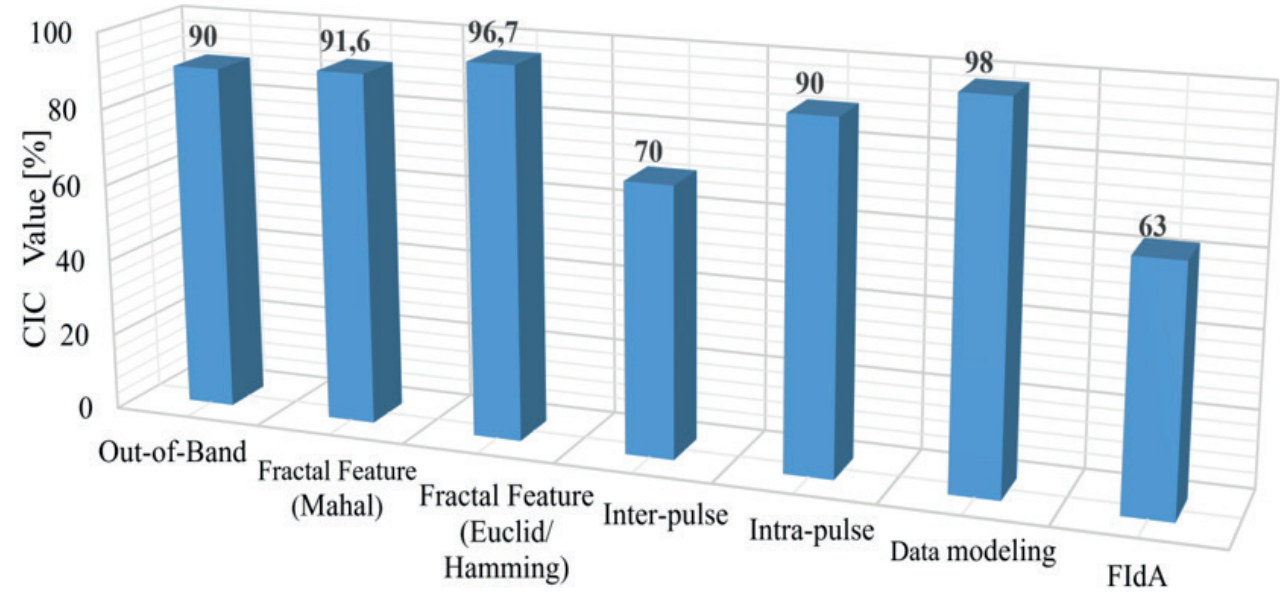

Fig. 6. Graphic illustration of CIC values for other RER methods 
the presented RER method based on multi-channel recognition system makes it possible to receive the CIC coefficient which equals $61.4 \%$. In order to increase the CIC coefficient value, in further works on RER use in SEI process, a common similarity matrix will be defined. This matrix will include the complexity of algorithms which are used in the RER method, estimation time, the requirements of the equipment platform and other requirements, which are significant in the process of quality estimation of a particular method. Thus, it will be possible to count automatically the similarities between vectors of basic measurable parameters for different radar copies of the same type. For ELINT systems working in real conditions, on a contemporary battlefield the automation of RER process and explicit identification of every single emitter in real time (with minimum time burden) are currently primary challenges for ELINT specialists.

\section{REFERENCES}

[1] X. Zhang, Y. Shi and Z. Bao, “A new feature vector using selected bispectra for signal classification with application in radar target recognition”, IEEE Trans. on Signal Proc. 49, 1875-1885 (2001).

[2] V.C. Chen and H. Ling, "Joint time-frequency analysis for radar signal and image processing”, IEEE Signal Proc. Mag. 16, 81-93 (1999).

[3] I. Jouny, E. D. Garber and R. L. Moses, "Radar target identification using the bispectrum: A comparative study", IEEE Trans. Aerosp. Electron. Syst. 31, 69-77 (1995).

[4] S. Chen and D. Zhang, "Robust image segmentation using FCM with spatial constraints based on new kernel-induced distance measure", IEEE Trans. on Systems, Man, and Cybernetics 34, 1907-1916 (2004).

[5] T. Heseltine, N. Pears and J. Austin, "Three-dimensional face recognition using combinations of surface feature map subspace components", Image and Vision Computing 26, 382-396 (2008).

[6] W.L. Melvin and J.R Guerci, "Knowledge-aided signal processing: a new paradigm for radar and other advanced sensors", IEEE Trans. Aerosp. Electron. Syst. 42, 983-996 (2006).

[7] J. Dudczyk and A. Kawalec, "Fast-decision identification algorithm of emission source pattern in database", Bull. Pol. Ac.: Tech. 63, 385-389 (2015).

[8] J. Roe and A. Pudner, "The real-time implementation of emitter identification for ESM", Signal Processing in Electronic Warfare, IEE Colloquium, London, 1-6 (1994).

[9] G.T. Capraro A. Farina, H. Griffiths and M.C. Wicks, "Knowledge-based radar signal and data processing: a tutorial review", IEEE Signal Proc. Mag. 23, 18-29 (2006).

[10] J. Roe, S. Cussons and A. Felthman, "Knowledge-based Signal Processing for Radar ESM Systems", IEEE Proc. F-Radar and Signal Processing. 137, 293-301 (1990).

[11] A. Aubry, A. Bazzoni, V. Carotenuto, A.De Maio and P. Failla, "Cumulants-based radar specific emitter identification", IEEE International Workshop on: Infrmation Forensics and Security (WIFS), (2011).

[12] J. Dudczyk, "Applying the radiated emission to the radioelectronic devices identification", Ph.D. Thesis, Warsaw University of Technology (2004), [in Polish].

[13] J. Dudczyk and A. Kawalec, "Identification of emitter sources in the aspect of their fractal features", Bull. Pol. Ac.: Tech. 61, pp. 623-628 (2013).
[14] G. Zhang, W. Jin and L. Hu, "Fractal feature extraction of radar emitter signals", Proc. Asia-Pacific Conf. on Environmental Electromagnetics CEEM 2003. pp. 161-164, 4-7 Nov. China, (2003).

[15] J. Dudczyk and A. Kawalec, "Fractal features of specific emitter identification", Acta Phys. Pol A. 124, 406-409 (2013).

[16] J. Dudczyk, J. Matuszewski and A. Kawalec, "Specific emitter identification based on an inter-pulses modulation of radar signal", Przegląd elektrotechniczny R.92, No 9/2016, 267-217 (2016).

[17] A. Kawalec and R. Owczarek, "Radar emitter recognition using intrapulse data", Proc. of the NordSec 2005 - The 10th Nordic Workshop on Secure IT-Systems, 444-457, Warsaw (2004) .

[18] A. Cohen, I. Daubechies, O.G. Guleryuz and M.T. Orchard, "On the importance of combining wavelet-based nonlinear approximation with coding strategies", IEEE Trans. on Information Theory 48, 1895-1921 (2002).

[19] J. P. D'Ales and A. Cohen, "Non-linear approximation of random functions", SIAM J. Appl. Math. 57 (2), 518-540 (1997).

[20] J. Friedman, "Multivariate adaptive regression splines", Ann. Statist. 19, (1), 1-141 (1991).

[21] L. Breiman, "Hinging hyperplanes for regression, classification, and function approximation", IEEE Trans. on Information Theory. 39, 999-1013 (1993).

[22] M. Ren, J. Cai, Y. Zhu and M. He, "Radar emitter signal classification based on mutual information and fuzzy support vector machines", Proc. of the Int. Conf. on Software Process, 1641-1646, Beijing (2008).

[23] I. Jouny, F.D. Garber and S.C. Ahalt, "Classification of radar targets using synthetic neural networks", IEEE Trans. Aerosp. Electron. Syst. 29, 336-344 (1993).

[24] N. Petrov, I. Jordanov and J. Roe, "Identification of radar signals using neural network classifier with low-discrepancy optimization", IEEE Congress on Evolutionary Computation, 2658-2664, Cancun (2013).

[25] V.C. Chen and H. Ling, "Joint time-frequency analysis for radar signal and image processing", IEEE Signal Proc. Magazine. 16 (2), 1681-93 (2002).

[26] L. Li, H.-B. Ji and L. Jiang, "Quadratic time-frequency analysis and sequential recognition for specific emitter identification", IET Signal Proc. 5, 568-574 (2011).

[27] Z. Yang, W. Qiu, H. Sun and A. Nallanathan, "Robust radar emitter recognition based on the three-dimensional distribution feature and transfer learning", Sensors 16, 289 (2016).

[28] J. Dudczyk, "Radar emission sources identification based on hierarchical agglomerative clustering for large data sets", Sensors J., 1-9 (2016).

[29] C.S. Shieh and C.T. Lin, "A vector neural network for emitter identification”, IEEE Trans. Ant. Prop. 50, 1120-1127 (2002).

[30] G. B. Willson, "Radar classification using a neural network", Proc of SPIE 1294, 200-210 (1990).

[31] J.-B. Yang and D.-L. Xu, "On the evidential reasoning algorithm for multiple attribute decision analysis under uncertainty", IEEE Trans. on Systems, Man, and Cybernetics-Part A: Systems and Humans. 32, 1083-4427 (2002).

[32] A. Kawalec, R. Owczarek and J. Dudczyk, "Data modeling and simulation applied to radar signal recognition", Molecular and Quantum Acoustics 26, 165-173 (2005).

[33] G. Latombe, E. Granger and F. Dilkes, "Fast learning of grammar production probabilities in radar electronic support", IEEE Trans. Aerosp. Electron. Syst. 46, 1262-1289 (2010).

[34] K. Copsey and A. Webb, "Bayesian gamma mixture model approach to radar target recognition", IEEE Trans. Aerosp. Electron. Syst. 39, 1201-1217 (2003). 